characteristics and environmental features of mass-shooting locations in US cities.

Methods Using Gun Violence Archive data, we identified all mass-shootings in the 10 US cities with the highest homicide rates from 2014-2017 $(n=214)$. We geocoded the event locations and used American Community Survey estimates to describe the sociodemographic characteristics of mass-shooting block groups with 6 indicators of structural disadvantage. We compared mass-shooting block group sociodemographic characteristics with each city's characteristics overall using Students t-tests. We assessed the environmental features of mass-shooting locations using Google Street View to code 60 elements of the visible environment.

Results Compared to overall city demographics, mass-shooting block groups had significantly higher rates of poverty $(28.0 \%$ vs. $35.1 \% ; \mathrm{p}=0.004)$, unemployment $(8.2 \%$ vs. $10.1 \%$; $\mathrm{p}=0.005)$, Black residents $(47.5 \%$ vs. $70.0 \% ; \mathrm{p}=0.012)$ and renter occupied units $(53.0 \%$ vs. $57.9 \%$; $\mathrm{p}=0.027)$, while percentage of college attendees/graduates $(55.8 \%$ vs. $30.2 \%$; $\mathrm{p}<0.0001)$, and median household income $(\$ 37,302$ vs. $\$ 31,313 ; p=0.009$ ) were significantly lower in mass-shooting block groups. Sixty-four percent of mass-shootings occurred in residential locations, and most locations demonstrated indicators of physical disorder. Average building conditions were graded as moderate; $37.4 \%$ of locations had buildings with broken/boarded windows and $78.3 \%$ had moderate to extreme littering. $94.3 \%$ and $93.1 \%$ of locations had no parks or playgrounds respectively.

Conclusions Mass-shootings in urban environments tend to occur in residential locations with significant structural disadvantage. The built environment of mass-shooting locations is characterized by blighted buildings and limited green spaces.

Contributions to Injury and Violence Prevention Science Discourse about mass shootings often excludes urban spaces. Structural disadvantage and modifiable environmental features are key targets for mass-shooting prevention in US cities.

\section{ASSOCIATED RISKS FOR FALLS AMONG ADULTS AGES 65 AND OLDER: INSIGHTS FROM THE NATIONAL HEALTH INTERVIEW SURVEY, 2015-2017}

Matthew Garnett, Holly Hedegaard, Merianne Spencer. Centers for Disease Control and Prevention

\subsection{6/injuryprev-2020-savir.124}

Statement of Purpose This analysis uses data from the National Health Interview Survey (NHIS), a national in-person survey of non-institutionalized adults, to examine risk factors among adults aged 65 and older who reported a fall.

Methods/Approach Data from the NHIS was used to identify older adults who experienced a fall-related injury in the 90 days prior to survey participation. Analysis was conducted using data from all older adults who reported a fall and on the subset of adults who had a fall that required emergency department (ED) treatment or hospitalization. The study used aggregate data from 2015-2017. Established methodologies to account for the complex sampling and survey design were employed to generate national estimates. Bivariate (X2 tests) and multivariate (logistic regression) analyses were conducted to examine differences by demographic characteristics and comorbid medical conditions.
Results During the 3-year period, there were 939 older adult survey participants who reported a fall in the past 90 days (national estimate $=1,020,779$ ). Of these, 534 were hospitalized or treated at an emergency department (ED) (national estimate $=589,251$ ). Falls were significantly associated $(p<0.05)$ with increasing age, being female, being white-non-Hispanic, and being widowed. After controlling for demographic factors, comorbid conditions that limited daily activities/functioning and were significantly associated with falls included: depression/anxiety, dementia, cancer, arthritis, diabetes, lung problems (asthma and other breathing conditions), and being obese. Similar findings were observed for severe falls that required hospitalization or ED treatment.

Conclusions The NHIS provides insight into the risk of falls among older adult populations. Disparities exist across demographic groups, and key co-morbidities may affect fall risk among older adults.

Significance/Contributions Studies examining falls among older adult populations are often limited to examining health outcomes or disease burden. This analysis adds contextual information on possible risk factors for falls using nationally representative survey data.

\section{FUN RIDE OR RISKY TRANSPORT: GOLF CART-RELATED INJURIES TREATED IN U.S. EMERGENCY DEPARTMENTS FROM 2007 THROUGH 2017}

Rebecca McAdams, Kyle Horvath, Kristin Roberts, Motao Zhu, Lara McKenzie. Center for Injury Research Policy at the Abigail Wexner Research Institute at Nationwide Children's Hospital

\subsection{6/injuryprev-2020-savir.125}

Purpose Golf cart-related injuries are a source of morbitiy for people of all ages, especially children, and their use has increased in recent years. However, design modifications or legislation to reduce the overall burden of these injuries have been nonexistent. This study sought to characterize the epidemiology of golf cart-related injuries treated in United States (U.S.) hospital emergency departments (EDs).

Methods/Approach We performed a retrospective analysis by using the National Electronic Injury Surveillance System data for patients of all ages treated in EDs (2007-2017) for a golf cart-related injury. Bivariate comparisons were conducted by using design-adjusted chi-square tests, and the strength of association was assessed by relative risks (RR) with corresponding 95\% confidence intervals (CIs). Injury rates over time were analyzed by using linear regression. We generated national estimates from 3567 cases.

Results From 2007-2017, an estimated $156 \quad 040 \quad$ (95\% $\mathrm{CI}=102$ 402-209 679) patients were treated in U.S. EDs for golf cart-related injuries. The average rate of traumatic brain injuries (TBIs) in children (1.62 per 100000 children) was more than three times that of TBIs in adults $(0.52$ per 100 000 adults; rate ratio $=3.12 ; 95 \% \mathrm{CI}=3.05-3.20) 1.46$ times that of TBIs in seniors (1.11 per $100 \quad 000$ seniors; 95\% $\mathrm{CI}=1.42-1.51)$. The injury rate in seniors increased significantly by $67.6 \%$ from 4.81 per 100000 seniors in 2007 to 8.06 per 100000 seniors in 2017 (slope $=0.096$; $\mathrm{p}=0.041$ ). Conclusions Golf cart use remains an important source of injury for people of all ages, especially children. As use continues to increase, it is unlikely that golf cart-related injuries 